\title{
Changes in Synaptic Morphology Accompany Actin Signaling during LTP
}

\author{
Lulu Y. Chen, ${ }^{1 *}$ Christopher S. Rex ${ }^{3 *}$ Malcolm S. Casale, ${ }^{2}$ Christine M. Gall, ${ }^{1,3}$ and Gary Lynch ${ }^{2}$ \\ Departments of ${ }^{1}$ Anatomy and Neurobiology and ${ }^{2}$ Psychiatry and Human Behavior, University of California, Irvine, California 92697-4292, and \\ ${ }^{3}$ Department of Neurobiology and Behavior, University of California, Irvine, California 92697-4550
}

\begin{abstract}
Stabilization of long-term potentiation (LTP) is commonly proposed to involve changes in synaptic morphology and reorganization of the spine cytoskeleton. Here we tested whether, as predicted from this hypothesis, induction of LTP by theta-burst stimulation activates an actin regulatory pathway and alters synapse morphology within the same dendritic spines. TBS increased severalfold the numbers of spines containing phosphorylated (p) p21-activated kinase (PAK) or its downstream target cofilin; the latter regulates actin filament assembly. The PAK/cofilin phosphoproteins were increased at $2 \mathrm{~min}$ but not $30 \mathrm{~s}$ post-TBS, peaked at 7 min, and then declined. Double immunostaining for the postsynaptic density protein PSD95 revealed that spines with high pPAK or pCofilin levels had larger synapses $(+60-70 \%)$ with a more normal size frequency distribution than did neighboring spines. Based on these results and simulations of shape changes to synapse-like objects, we propose that theta stimulation markedly increases the probability that a spine will enter a state characterized by a large, ovoid synapse and that this morphology is important for expression and later stabilization of LTP.
\end{abstract}

Key words: synaptic plasticity; p21-activated kinase; cofilin; dendritic spines; postsynaptic density; phosphorylation

\section{Introduction}

Ultrastructural studies indicate that long-term potentiation (LTP) is accompanied by a change in synaptic morphology, but there is considerable uncertainty as to the nature of the adjustment. Several experiments suggest that a simple change in size and shape is involved (Fifkova and Van Harreveld, 1977; Lee et al., 1979, 1980; Lang et al., 2004; Nagerl et al., 2004; Zhou et al., 2004), whereas others point to a perforation or splitting of the synaptic region (Jones and Calverley, 1991; Muller et al., 2000). These results provide a plausible explanation for the increase in synaptic currents that constitutes LTP expression because of growing evidence that AMPA receptor number scales with the size of the synapse (Nusser et al., 1998; Takumi et al., 1999; Racca et al., 2000; Ganeshina et al., 2004). Possibly related to these morphological observations are light microscopic results showing that intense synaptic activity modifies spine actin networks in cultured cells (Fischer et al., 1998; Matus et al., 2000; Okamoto et al., 2004). Synaptically driven polymerization has been described for the adult hippocampus (Fukazawa et al., 2003) with recent work, using an in situ F-actin labeling procedure, demonstrating the effect within individual spine heads (Lin et al., 2005). Extensions to the latter work indicate that polymerization is essential to

\footnotetext{
Received Jan. 15, 2007; revised April 11, 2007; accepted April 12, 2007.

This work was supported by National Institute of Neurological Disorders and Stroke Grants NS051823, NS45260, and NS37799. C.S.R. was supported by Grant AG00358 from the National Institute on Aging. Use of the Bio-Rad Radiance 2000 confocal microscope system was provided by the University of California, Irvine Institute for Brain Aging and Dementia. We thank Michael Mateling for assistance with image analysis.

*L.Y.C. and C.S.R. contributed equally to this work.

Correspondence should be addressed to Christine M. Gall, Gillespie Neuroscience Research Facility, University of California, Irvine, CA 92697-4292. E-mail: cmgall@uci.edu.

D0I:10.1523/JNEUROSCI.0164-07.2007

Copyright $\odot 2007$ Society for Neuroscience $\quad$ 0270-6474/07/275363-10\$15.00/0
}

the stabilization, rather than the expression, of LTP (Kramar et al., 2006).

The above findings suggest that the theta-burst stimulation (TBS) used to induce synaptic potentiation initiates changes in synaptic anatomy while triggering reorganization of the spine cytoskeleton, with these effects respectively providing for expression and stabilization of LTP. Although this hypothesis accounts for a diverse array of findings, there have been no tests of the essential prediction that TBS rapidly alters actin dynamics and produces a change in synapse morphology within the same spine. The present studies addressed this issue. We first asked whether TBS increases phosphorylation of p21-activated kinase (PAK) and its downstream effector cofilin in spines within adult hippocampal slices. Phosphorylation of cofilin is one of the routes whereby Rho GTPases regulate actin filament assembly (Juliano et al., 2004; Carlisle and Kennedy, 2005). Moreover, the PAK/ cofilin pathway is engaged in various cell systems by two receptor classes, integrins and neurotrophin receptors (Gehler et al., 2004; Juliano et al., 2004; Soule et al., 2006), that play prominent roles in theta-induced actin polymerization (Kramar et al., 2006) and spine morphogenesis (Penzes et al., 2003; Boda et al., 2004; Hayashi et al., 2004; Boda et al., 2006). There are thus multiple reasons for assuming that activation of the PAK/cofilin pathway is part of the sequence of events leading from TBS to cytoskeletal reorganization.

In addition to helping to explain how structural changes are initiated during LTP induction, positive results in the PAK/cofilin experiments could open the way to a test of whether thetainduced changes in actin dynamics within spines are associated with changes in synapse morphology. Here we report results that accord with this prediction but that also call for a probabilistic version of the broad hypothesis (for review, see Lynch and 
Baudry, 1984; Yuste and Bonhoeffer, 2001) that the production of stable LTP involves the generation of a new synaptic architecture.

\section{Materials and Methods}

All animal procedures were conducted in accordance with the National Institutes of Health Guide for the Care and Use of Laboratory Animals and with protocols approved by the Institutional Animal Care and Use Committee of the University of California, Irvine.

Electrophysiology. Transverse hippocampal slices (350 $\mu \mathrm{m}$ thick from midseptotemporal region) were prepared from young adult (1-2 months of age) male Sprague Dawley rats (Charles River Laboratories, Wilmington, MA) as previously described (Rex et al., 2005) and maintained in an interface recording chamber $\left(95 \% \mathrm{O}_{2} / 5 \% \mathrm{CO}_{2}\right)$ perfused $(60-70 \mathrm{ml} / \mathrm{h}$, $31 \pm 1^{\circ} \mathrm{C}$ ) with artificial CSF (aCSF) containing the following (in $\mathrm{mm}$ ): $124 \mathrm{NaCl}, 3 \mathrm{KCl}, 1.25 \mathrm{KH}_{2} \mathrm{PO}_{4}, 3.4 \mathrm{CaCl}_{2}, 2.5 \mathrm{MgSO}_{4}, 26 \mathrm{NaHCO}_{3}$, and 10 dextrose (Rex et al., 2005, 2006). Slices were allowed to equilibrate in the recording chamber for $2-3 \mathrm{~h}$ before recordings or other treatments were initiated. Unless otherwise noted, chemicals were purchased from Sigma (St. Louis, MO).

Recordings of field EPSPs (fEPSPs) were collected from CAlb stratum (str.) radiatum using a single glass pipette $(5 \mathrm{M} \Omega, 2 \mathrm{M} \mathrm{NaCl})$. Bipolar stimulation (twisted nichrome wires) was delivered alternately at two sites (CAla and CAlc) in the apical Schaffer-commissural projections (Kramar et al., 2006). The amplitude of the fEPSPs were set to $30-40 \%$ of maximum. Low-frequency baseline stimulation was delivered at $0.05 \mathrm{~Hz}$ for 20-30 min as described previously (Rex et al., 2005). Slopes and amplitudes of fEPSPs were measured from digitized files (NacGather 2.0; Theta Burst, Irvine, CA) and normalized to the last $10 \mathrm{~min}$ of the baseline period. Synaptic potentiation was induced using TBS (10 bursts of four pulses at $100 \mathrm{~Hz}$ separated by $200 \mathrm{~ms}$ ) (Larson et al., 1986). TBS was delivered to the two stimulation electrodes within $5 \mathrm{~s}$ of each other, and slices were collected at time points described below; control slices continued to receive baseline stimulation for an equivalent period.

Bath infusion of 6,7-dinitroquinoxaline-2,3-dione (DNQX; Tocris Bioscience, Ellisville, MO) or DL-2-amino-5-phosphonovaleric acid (APV; Tocris Bioscience) was performed via a second infusion line attached to the main input line. DNQX $\left(20 \mathrm{~mm}\right.$, stored at $\left.-20^{\circ} \mathrm{C}\right)$ and APV $\left(25 \mathrm{~mm}, 4^{\circ} \mathrm{C}\right)$ stocks were prepared in dimethylsulfoxide and doubly distilled $\mathrm{H}_{2} \mathrm{O}$, respectively, and diluted to working concentrations in aCSF before experimentation.

Immunocytochemistry. The following primary antisera and dilutions were used: anti-PAK3 (1:500; catalog \#06-902; Upstate, Charlottesville, VA), anti-PAK1 (1:250; catalog \#2602; Cell Signaling Technology, Danvers, MA), anti-phospho-PAK ( $\mathrm{pS} 141$ ), which recognizes the conserved phosphorylation site on PAKs 1, 2, and 3 (1:100; catalog \#44-940G; Biosource, Camarillo, CA), anti-cofilin (1:250; catalog \#ACFL02; Cytoskeleton, Denver, CO), anti-phospho-cofilin (pS3; 1:100; catalog \#12866; Abcam, Cambridge, MA), and anti-postsynaptic density 95 (PSD95; 1:1000; catalog \#MA1-045; Affinity Bioreagents, Golden, CO). The antiPSD95 is a mouse monoclonal; all other primary antisera were raised in rabbit. For preparation of hippocampal slices not receiving electrical stimulation, slices were prepared as described for electrophysiology and maintained in oxygenated aCSF for 2-3 h. For analysis of immunolabeling after electrophysiological recording, slices were fixed in $4 \%$ paraformaldehyde in $0.1 \mathrm{~m}$ sodium phosphate buffer (PB), pH 7.2, for 12-16 h, cryoprotected in $20 \%$ sucrose/PB for $2 \mathrm{~h}$ at $4^{\circ} \mathrm{C}$, sectioned on a freezing microtome at $20 \mu \mathrm{m}$, and then mounted onto Fisher Superfrost Plus slides (Fisher Scientific, Pittsburgh, PA). Sections were incubated ( $40 \mathrm{~h}$, $4^{\circ} \mathrm{C}$ ) in one or more primary antisera in $0.1 \mathrm{M} \mathrm{PB}$ containing $4 \%$ bovine serum albumin and $0.3 \%$ Triton X-100 (PBT). Slides were washed in PB $(3 \times 10 \mathrm{~min})$, incubated (45 $\mathrm{min}$ at room temperature) with Texas Red anti-rabbit IgG or fluorescein anti-mouse IgG (1:200; Vector Laboratories, Burlingame, $\mathrm{CA}$ ) in $\mathrm{PBT}$, rinsed in $\mathrm{PB}$ and coverslipped with Vectashield (Vector Laboratories).

Control tissue was processed by the same procedures but with exclusion of one, or the other, primary antisera from the initial incubation step: under these conditions, the only labeling observed was associated with the primary antibody included in the initial incubation, thereby demonstrating the species specificity of secondary antisera.

Confocal microscopy and measurement of immunoreactive puncta. Laser-scanning confocal microscopy was performed using the Bio-Rad (Hercules, CA) Radiance 2000 Laser Scanning System using a 60× [1.4 numerical aperture (NA)] Plan Apo objective. Optical sections $(1.0 \mu \mathrm{m})$ were scanned at $4 \times$ zoom in a $512 \times 512$ pixel format. Image montages covering a $205 \times 205 \mu \mathrm{m}$ area were collected from the zone between the two stimulation electrodes containing potentiated synapses (Shimono et al., 2002). The sample field $\left(3126 \mu \mathrm{m}^{2}\right)$ selected for puncta quantification was converted to grayscale, and intensity levels were scaled to values determined for each experiment (Photoshop CS, version 8.0; Adobe Systems, San Jose, CA) to visualize low-intensity labeling. Analysis was conducted blind to sample identity on batches of slices that had been sectioned and stained together. In-house built software (Lin et al., 2005; Kramar et al., 2006) was used to count and determine the area of labeled puncta within the size range of spines and synapses. Briefly, intensity thresholds ( 8 bits/pixel) were applied to identify punctate, spine-like structures at varying levels of label intensity within a range that reliably counted puncta. Thresholds, in practice, had to be placed well above the level at which diffuse, background fluorescence appeared. Lightly labeled elements were therefore lost to the analysis. As a consequence, numbers of a given anatomical structure (i.e., incidence per unit area) as estimated from immunostained sections are significantly smaller than the values recorded with electron microscopy.

Pixel values for each image were normalized to reduce the impact of background intensity differences across the image, binarized using each intensity threshold, and finally cleaned by erosion and dilation filtering (Jain, 1984). Counts from two serial sections were averaged to produce a representative value for each tissue slice. Identified objects $<0.04$ and $>1.2 \mu \mathrm{m}^{2}$ were excluded from analysis. Colocalization of immunostaining for phosphorylated (p) cofilin (pCofilin) and pPAK with that for PSD95 was determined automatically (Matlab; Mathworks, Natick, MA) using center coordinates and radius of each labeled object to determine overlap.

Widefield microscopy and three-dimensional reconstruction. Widefield photomicrographs of immunostained tissue sections were acquired with a Leica DM6000 B microscope (Leica Microsystems, Bannockburn, IL) using a $63 \times$ Plan Apo (1.4 NA) objective. $Z$-series ( $0.2 \mu \mathrm{m}$ step) images were deconvolved by iterative restoration using Volocity 4.0 Restoration software (Improvision, Lexington, MA). Three-dimensional reconstructions of the target field were generated and qualitatively analyzed for overlap between phosphoprotein- (pPAK or pCofilin) and PSD95immunoreactive elements.

Statistical analyses. Analysis of electrophysiological recordings was performed by repeated-measures ANOVA of the final $5 \mathrm{~min}$ of experimental recording. Statistical significance of puncta counts was determined by $t$ test or ANOVA followed by Tukey's HSD post hoc test. Population counts for these experiments represent numbers of slices. Group puncta size distributions were compared using the Kolmogorov-Smirnov $Z$ test. All measures are reported as group means \pm SD in text and as means \pm SEM in figures.

\section{Results}

\section{Theta-burst stimulation causes time-dependent phosphorylation of PAK}

The PAK family is composed of six highly conserved GTPasedriven serine/threonine kinases that regulate actin dynamics through parallel pathways that use cofilin, myosin light chain kinase, and other effectors (Hayashi et al., 2004; Juliano et al., 2004; Boda et al., 2006). Two PAK isoforms (PAK3 and PAK1) have been localized to synapses and implicated in spine morphogenesis in the immature hippocampus (Penzes et al., 2003; Boda et al., 2004), but there are as yet no descriptions of the synaptic localization of PAK in adult brain. Accordingly, sections from hippocampal slices were processed for immunocytochemical localization of PAK3 and PAK1 in CA1 str. radiatum using laserscanning confocal microscopy. PAK3 immunoreactivity was 

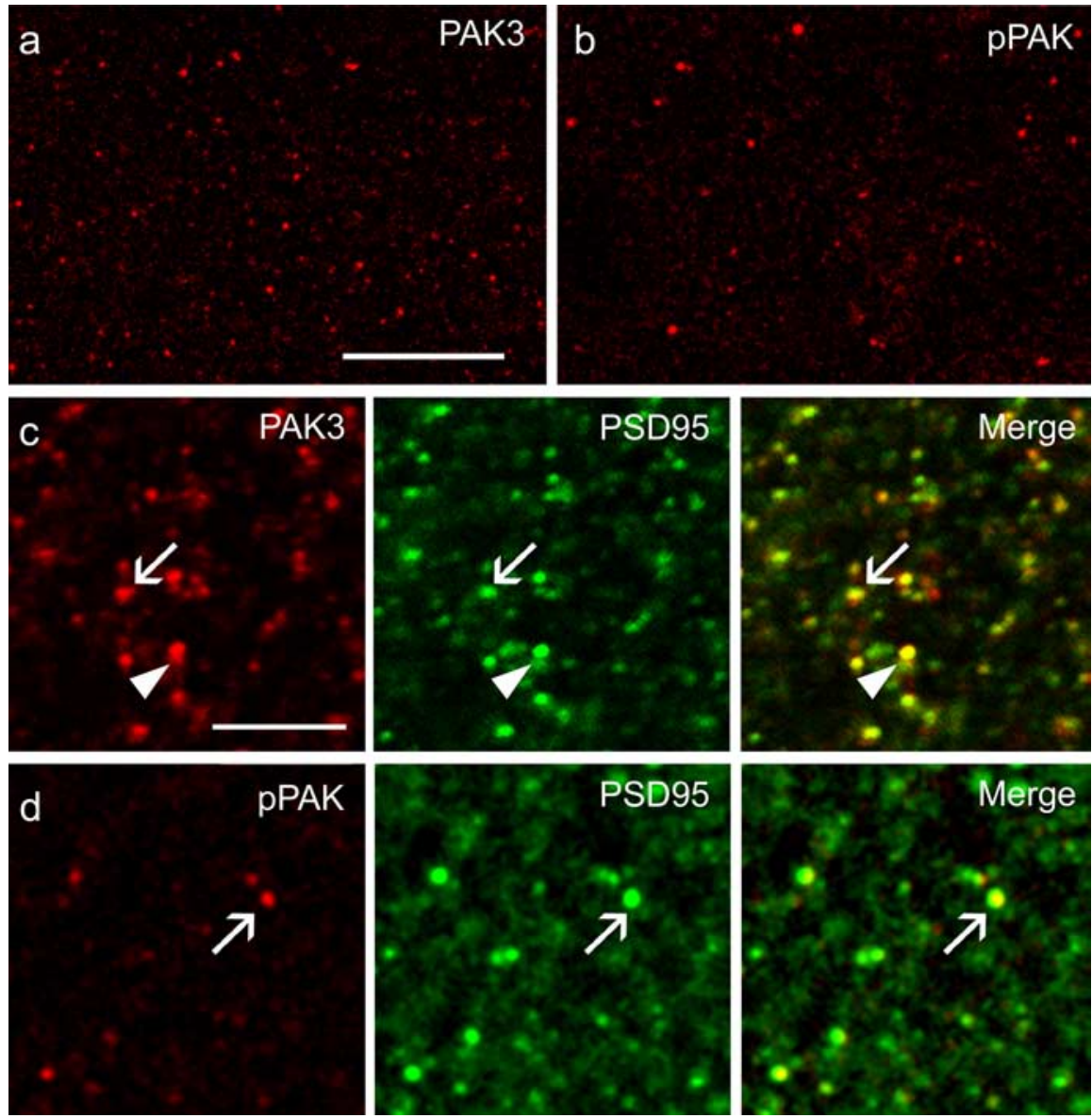

Figure 1. Immunostaining for total and pPAK proteins in stratum radiatum of hippocampal field CA1. $\boldsymbol{a}, \boldsymbol{b}$, Laser-scanning confocal micrographs show the distributions of immunolabeling for PAK3 $(\boldsymbol{a})$ and pPAK $(\boldsymbol{b})$. The CA1 cell body layer is to the right (data not shown) in each micrograph. Note that dense PPAK-IR puncta are much less numerous than the comparably sized structures labeled for total PAK3 immunoreactivity. Scale bar, $10 \mu \mathrm{m}$. c, Confocal micrographs illustrate the distributions of PAK3 (red), PSD95 (green), and overlapped immunolabeling (yellow in Merge). PAK3-IR puncta were typically associated with dense labeling for PSD95, with the latter either capping (arrow) or overlapping (arrowhead) the PAK3 immunoreactivity. Scale bar, 5 $\mu \mathrm{m}$. $\boldsymbol{d}$, Confocal micrographs show profiles labeled with anti-pPAK (red) and anti-PSD95 (green) and the overlap of the two (yellow in Merge). The majority of pPAK-IR profiles were associated with PSD95 immunoreactivity. Scale bar, $5 \mu \mathrm{m}$.

densely concentrated in puncta corresponding in size, shape, and distribution to dendritic spines and was largely absent from dendritic shafts and axonal processes (Fig. 1a). The spine identity of the PAK3-positive $\left({ }^{+}\right)$puncta was confirmed by double immunostaining for PSD95, a synaptic scaffold protein that is highly enriched in glutamatergic synapses (Hunt et al., 1996). PSD95 is reported to be present at all asymmetric synapses (Petersen et al., 2003), uniformly distributed across the full extent of the synaptic active zone (Valtschanoff and Weinberg, 2001; Petersen et al., 2003; Sassoe-Pognetto et al., 2003), and not found in presynaptic terminals (Hunt et al., 1996) or inhibitory synapses (Aoki et al., 2001). As expected from these points, the field of PSD95 immunogold labeling corresponds well with the size of its associated postsynaptic density (Aoki et al., 2001).

The PAK3 immunoreactivity was typically partially colocalized with, or capped by, the $\mathrm{PSD} 95^{+}$postsynaptic density (Fig. 1c). Immunostaining for PAK1 was basically the same as that for PAK3, although less abundant (data not shown). As expected from these results, labeling with antisera to PPAK (which recognized the conserved initial autophosphorylation site on PAKs 1, 2, and 3) (Chong et al., 2001) was localized almost exclusively in PSD95 $^{+}$spine-like profiles (Fig. $1 d$ ). However, the number of densely labeled pPAK-immunoreactive (-IR) puncta was much smaller than the number of total PAK-IR puncta (Fig. $1 a, b)$. Counts made with a computerized system provided estimates of $18.3 \pm 2.7$ puncta/100 $\mu \mathrm{m}^{2}$ for PAK and $1.6 \pm 1.6$ puncta/100 $\mu \mathrm{m}^{2}$ for pPAK (means \pm SDs).

Figure 2 summarizes the number of $\mathrm{pPAK}^{+}$spine-like profiles present in tissue collected at various times after the delivery of a single train of TBS to each of two collections of Schaffer-commissural projections innervating the analyzed dendritic field (proximal str. radiatum of field CA1b). Evoked responses recorded from this zone (Fig. 2a) confirmed that theta stimulation caused an immediate and dramatic potentiation [traces from slices collected at $2 \mathrm{~min}$ or $7 \mathrm{~min}$ post-TBS (Fig. 2a)] that stabilized over the subsequent 15-30 $\mathrm{min}$. A pronounced increase in the number of densely pPAK-IR spine-like puncta was apparent in slices collected 2-7 min post-TBS $\left[4.3 \pm 1.7\right.$ spines $/ 100 \mu \mathrm{m}^{2}$ (Fig. 2c)] compared with controls [1.5 \pm 1.9 (Fig. 2b)]. This effect was delayed in onset, because it was not detected at $30 \mathrm{~s}$ post-TBS $\left(1.2 \pm 1.0 \mathrm{puncta} / 100 \mu \mathrm{m}^{2}\right)$, and transient, in that slices collected at 15-30 min post-TBS $(1.3 \pm 1.8)$ did not have a reliably greater than normal number of pPAK $^{+}$puncta (Fig. $2 d$ ). The overall effect of TBS was significant (ANOVA; $p=0.024)$, as were the differences between the 2-7 min group and controls or slices collected at 15-30 min $(p<0.05)$. The numbers of PSD95-IR structures in the same sample fields (Fig. 2e) were not influenced by TBS (ANOVA; $p=0.37$ ). Similarly, antibodies against total PAK3labeled equal numbers of puncta within str. radiatum of control $(19.1 \pm 1.0)$ and TBS-activated slices (18.3 \pm 2.7 ; two-tailed $t$ test, $p=0.59$ ). These results are consistent with evidence that afferent stimulation increases PPAK in spines of dissociated neurons (Hayashi et al., 2004) and confirm the experimental prediction that LTP induction is associated with a rapid and relatively short-lasting activation of the PAK signaling pathway.

Although the relative effects of TBS were large (Fig. 2), the absolute numbers of synapses involved were small. Serial section electron microscopy indicates that $\sim 250$ synapses $/ 100 \mu \mathrm{m}^{3}$ are found in str. radiatum of the adult rat hippocampus (Fiala and Harris, 2001) with $>95 \%$ of these being located on spines. Because the optical sections used in the present analyses were $1 \mu \mathrm{m}$ thick, the values described in Figure 2 represent per $100 \mu \mathrm{m}^{3}$ values. Thus, the observed increase of 3.2 puncta (control vs $2-7$ min) would constitute a shift of only $\sim 1.3 \%$ of the total immunolabeled spine population into a $\mathrm{PPAK}^{+}$state. (It is noteworthy that the number of immunolabeled spines counted in our analysis is undoubtedly an underestimate of the total numbers of spines within the sample field because antibodies do not achieve full tissue penetration, and many lightly labeled spines will fall 
into the intensity range at which visual noise begins to intrude on the images.)

The above results demonstrate that TBS markedly increases the number of $\mathrm{pPAK}^{+}$profiles in the dendritic zone containing the potentiated synapses (i.e., the proximal str. radiatum) but do not establish the regional specificity of the effect. Accordingly, we counted $\mathrm{pPAK}^{+}$profiles in the str. lacunosum-moleculare $(<30$ $\mu \mathrm{m}$ from the hippocampal fissure), a lamina that does not receive Schaffercommissural input. The size of the assayed regions and the method for acquiring the images were the same as used for the potentiated zone; slices were collected 2-7 min post-TBS. Counts of $\mathrm{pPAK}^{+}$puncta were not significantly different for control slices $\left(0.5 \pm 0.4\right.$ puncta/ $\left.100 \mu \mathrm{m}^{2}\right)$ versus those receiving TBS $(0.3 \pm 0.1$ puncta $/ 100$ $\mu \mathrm{m}^{2}$; two-tailed $t$ test, $p=0.60, n=$ 6/group), thereby demonstrating that effects of theta stimulation on $\mathrm{pPAK}^{+}$ spine-like puncta are lamina specific.

\section{Phosphorylation of cofilin}

Cofilin accelerates the removal of monomers from the sharp end of actin filaments. This activity is blocked during phosphorylation by LIM kinase, a PAK effector; PAK activation thus results in suppression of cofilin activity and the creation of circumstances favorable for actin cytoskeletal assembly (Carlisle and Kennedy, 2005). Confocal and electron microscopic studies have shown that cofilin is not associated with presynaptic elements and concentrates in dendritic spines in the adult hippocampus (Racz and Weinberg, 2006). We confirmed this localization. Cofilin immunoreactivity was present in large numbers of spine-like puncta scattered throughout the CA1 apical dendritic field (Fig. 3a); counterstaining with antisera to PSD95 confirmed that the great majority of these structures contain postsynaptic densities. In contrast, very few puncta $(0.3 \pm$ $0.3 / 100 \mu \mathrm{m}^{2}$ ) contained high concentrations of pCofilin immunoreactivity and, as with the total protein, these were usually associated with PSD95 (Fig. 3b). Inspection of the two markers at high magnification (Fig. $3 c$ ) indicated that the pCofilin ${ }^{+}$structures, as with $\mathrm{pPAK}^{+}$puncta, were partially colocalized with, or capped by, PSD95 immunostaining. To more fully examine this spatial relationship, we used restorative deconvolution analysis (0.2 $\mu \mathrm{m} \mathrm{z}$-step), with images acquired by widefield microscopy, followed by serial reconstruction using Volocity 4 Restoration software (Fig. 3d). This technique confirmed that the overlap observed in two dimensions accurately depicts partial colocalization. (supplemental movies 1, 2, available at www.jneurosci.org as supplemental material). In addition, qualitative threedimensional examination of double-labeled puncta revealed that most PSD95-IR puncta were ellipsoid in shape, whereas pCofi$\mathrm{lin}^{+}$structures were more rounded, shapes that are consistent with PSD95 localization in the synaptic scaffold and pCofilin extending farther into the head of the spine.

Hippocampal slices receiving TBS exhibited a dramatic, timedependent increase in numbers of spine-like structures contain- ing dense concentrations of pCofilin immunoreactivity in the dendritic zone innervated by stimulated afferents (Fig. 3e). A more than sixfold increase in pCofilin ${ }^{+}$puncta was counted in slices collected from 2 to 7 min post-TBS $(1.9 \pm 1.5$ puncta/100 $\mu \mathrm{m}^{2}$ ) compared with control slices receiving only low-frequency stimulation $(0.3 \pm 0.3 ; p=0.008$, ANOVA, followed by Tukey's HSD). As was the case for pPAK, the number of pCofilin ${ }^{+}$puncta was not significantly different from control values in slices fixed $30 \mathrm{~s}(0.2 \pm 0.1 ; p=0.99)$ or $15-30 \mathrm{~min}(0.8 \pm 1.0 ; p=0.67)$ post-TBS. The overall effect of stimulation was highly significant (ANOVA; $p=0.002$ ). Analysis of double labeling for PSD95 again indicated that TBS did not influence numbers of PSD95 ${ }^{+}$ puncta in the sample field (ANOVA; $p=0.23$ ). Slices processed for localization of total cofilin immunoreactivity at $7 \mathrm{~min}$ postTBS similarly showed no effect of stimulation on numbers of cofilin ${ }^{+}$puncta $\left(10.2 \pm 1.38\right.$ puncta $/ 100 \mu \mathrm{m}^{2}$ vs $11.0 \pm 1.68$ for $7 \mathrm{~min}$ post-TBS vs controls, respectively; $p=0.48$ ). These data confirm in detail the previous conclusion that theta stimulation, after a brief delay, activates the PAK/cofilin pathway within the 5-10 min poststimulation period during which LTP undergoes a rapid phase of consolidation.

The sample sizes for the $2-7 \mathrm{~min}$ groups in the individual experiments described above were not sufficient to test whether slices collected at $2 \mathrm{~min}$ had increased numbers of spines in which the PAK/cofilin pathway had been engaged. We therefore combined cases for the separate markers (anti-pPAK, anti-pCofilin) of the signaling cascade at each time point by converting their values and those of the controls, to $Z$-scores using the means and 

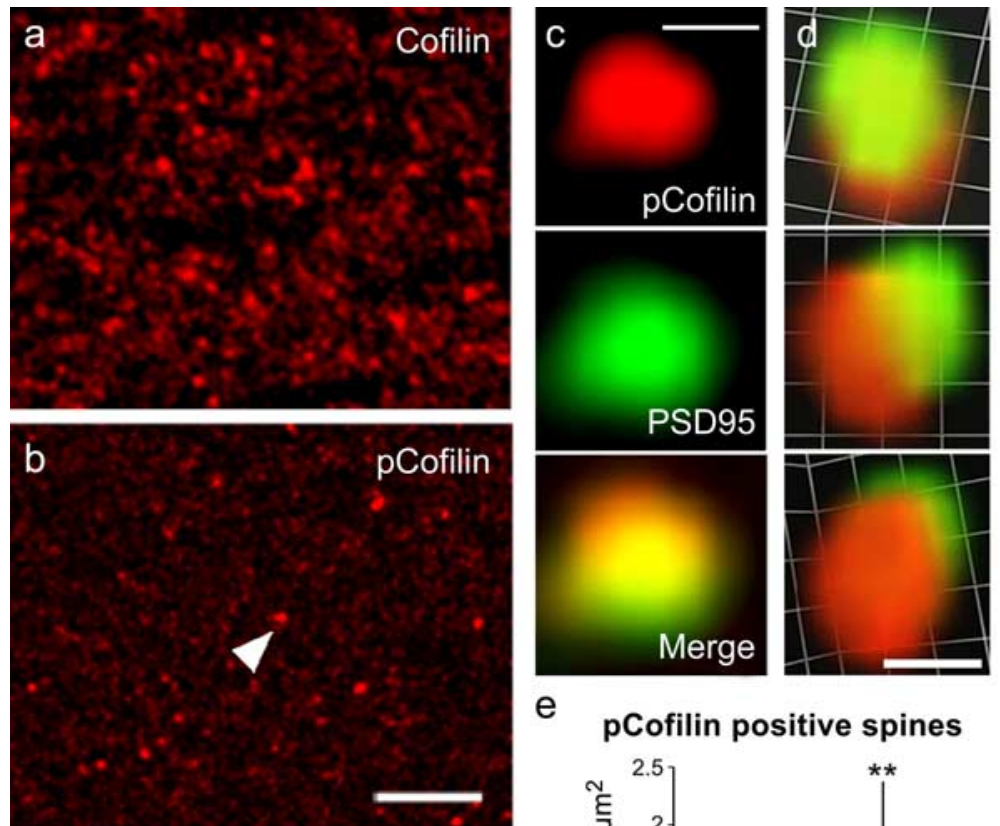

e
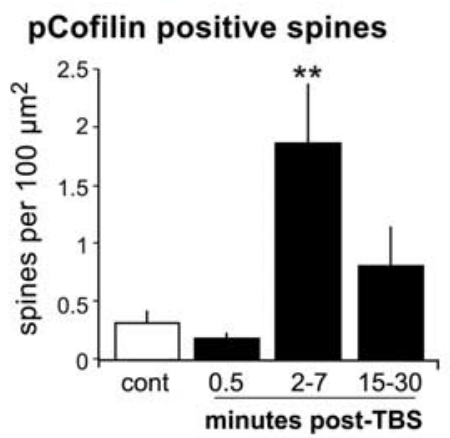

f

pPAK \& pCofilin positive spines

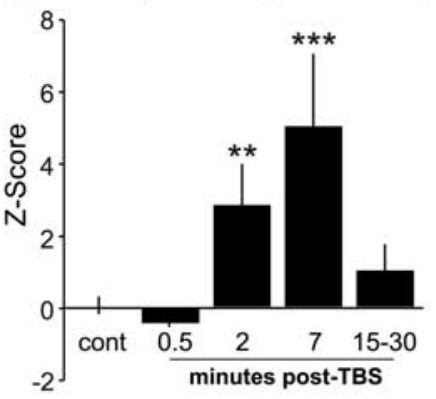

Figure 3. Effects of theta-burst stimulation on the number of spines with high concentrations of pCofilin. $\boldsymbol{a}$, Laser confocal micrograph shows immunolabeling for total cofilin in the proximal CA1 str. radiatum. Scale bar, $5 \mu \mathrm{m}$. $\boldsymbol{b}$, Micrographs showing immunostaining for pCofilin, PSD95, and the merged image of the two for the same field of CA1 str. radiatum. The pCofilin immunoreactivity (red) was localized to discrete structures that fell within the size range expected for dendritic spines. These were much less numerous than the comparable profiles $(\boldsymbol{a})$ labeled by antisera against total cofilin. Comparison of the three panels in $\boldsymbol{b}$ shows that a subpopulation of the numerous PSD95-IR structures (green) also contained pCofilin immunoreactivity (evident as yellow puncta in the right Merge panel) and that the majority of pCofilin-IR structures were associated with concentrations of PSD95 immunoreactivity. Scale bar, $5 \mu \mathrm{m}$. c, Enlargement of the structure indicated by the arrowhead in $\boldsymbol{b}$ illustrates the spatial relationship of areas occupied by pCofilin (red) and PSD95 (green) immunoreactivities and the extent to which the two overlap (yellow at bottom). Scale bar, $0.25 \mu \mathrm{m}$. $\boldsymbol{d}$, Three-dimensional reconstruction of immunolabeling for pCofilin (red) and PSD95 (green) structures after wide-field microscopic acquisition at $0.2 \mu \mathrm{m} z$-steps followed by restorative deconvolution: the adjacent but distinct localizations of pCofilin and PSD95 immunoreactivities are evident. The images are successive $90^{\circ}$ rotations (from top to bottom). Scale bar, $0.5 \mu \mathrm{m}$. e, Slices were collected 30 s, 2-7 min, or 15-30 min after the delivery of a single train of TBS to the Schaffer-commissural projections and processed for pCofilin immunostaining. Controls (cont; open bar) received baseline stimulation. The marked increase in pCofilin-IR puncta at $2-7$ min was highly significant ( ${ }^{* *} p=0.008$, Tukey's HSD) relative to controls and 30 s post-TBS groups ( $n=8$ for cont and $2-7 \mathrm{~min}, n=9$ for 0.5 and $15-30 \mathrm{~min}$ ). These results provide a close replication of those obtained with pPAK.f, Spine counts were combined within the various groups (control, 30 s post-TBS, etc.) from the separate pPAK and pCofilin experiments. Z-scores were calculated using the means and SDs for the control groups in each experiment; thus, each slice value was expressed as the difference from the mean of the control slices divided by the SD for the control slices. The planned comparisons of 2 and 7 min versus control was highly significant [ANOVA, $p=0.003,{ }^{* *} p=0.003$, and ${ }^{* * *} p=0.0009$ for $2(n=7)$ and $7(n=6)$ min, respectively].
SDs of the pPAK and pCofilin control groups (Fig. $3 f$ ). This resulted in groups of 16 control slices and 7 slices collected 2 min post-TBS. A significant increase in labeled puncta was evident in the latter group ( $p=0.003$ ) as well as at $7 \mathrm{~min}$ postTBS ( $n=6 ; p=0.0009$, two-tailed $t$ test). The overall effect of time for the combined groups was also highly significant $(p=$ 0.003 , ANOVA). It thus appears that the phosphorylation event begins between 30 and $120 \mathrm{~s}$ and continues for up to $420 \mathrm{~s}$ after delivery of theta stimulation.

The effects of TBS on pCofilin ${ }^{+}$puncta were blocked by application of the selective NMDA receptor antagonist (APV) at $50 \mu \mathrm{M}$. The APV infusion was initiated 30 min before stimulation and continued until the slices were collected at $7 \mathrm{~min}$ postTBS. The antagonist blocked LTP $(p>$ 0.8 for baseline vs the period from 2 to 7 min post-TBS; repeated-measures ANOVA) and completely eliminated TBSinduced increases in the number of $\mathrm{pCo}-$ filin ${ }^{+}$puncta (control stimulation, $0.55 \pm$ 0.34 puncta $/ 100 \mu \mathrm{m}^{2}, n=5$; 7 min postTBS in the presence of APV, $0.47 \pm 0.43$ puncta $/ 100 \mu \mathrm{m}^{2}, n=6 ; p=0.90$ vs control, two-tailed $t$ test).

\section{Phosphorylation in the PAK/cofilin pathway is associated with enlarged synapses}

Confirmation that theta-burst stimulation activates the PAK/cofilin cascade opened the way for tests of whether changes in actin dynamics within individual spines are, as proposed, associated with morphologically distinct synapses. Labeled PSD95 ${ }^{+}$synapses located atop pPAK or pCofilin-immunopositive puncta appeared larger than neighboring contacts in survey micrographs of the CA1 sampling field at $7 \mathrm{~min}$ post-TBS (Fig. $4 a$ ). Quantitative analyses confirmed this impression. As expected from earlier counts, the number of $\mathrm{pPAK}^{+}$puncta was substantially greater at $2-7 \mathrm{~min}$ post-TBS than in control slices; the synapses on these $\mathrm{pPAK}^{+}$puncta (at 2-7 min postTBS) had a mean PSD95 ${ }^{+}$area of $0.55 \pm$ $0.12 \mu \mathrm{m}^{2}$, whereas, in the same fields, the area value for the much more numerous synapses single labeled for PSD95 immunoreactivity was $0.30 \pm 0.05 \mu \mathrm{m}^{2}(p=$ 0.00007 , paired $t$ test for $n=8$ slices) (Fig. $4 b)$. Surprisingly, this impressive difference was not unique to the $2-7$ min postTBS group: synapses on the occasional pPAK $^{+}$puncta were $\sim 70 \%$ larger than their neighbors at all experimental time points (30 s and $15 \mathrm{~min}$ post-TBS) and in control (no TBS) slices $(p=0.001$ in all 
cases). It thus appears that induction of LTP in a subgroup of spines in the sampling region converts those spines into a state that is present, but only rarely, under control conditions.

The same pattern of quantitative results was obtained in a second series of slices that were processed for pCofilin immunostaining (Fig. 4b). The mean number of PSD95 ${ }^{+} /$pCofilin $^{+}$puncta was approximately fivefold greater at 2-7 $\mathrm{min}$ post-TBS than in control tissue. The PSD95 $^{+}$synapses associated with pCofilin immunostaining were substantially larger than was the case for synapses that were $\mathrm{PSD}^{+}{ }^{+}$alone $\left(0.44 \pm 0.06 \mu \mathrm{m}^{2}\right.$ vs $0.28 \pm 0.03 \mu \mathrm{m}^{2}$, respectively; $p=$ 0.00001 , paired $t$ test for eight slices). It was also the case that synapses associated with the occasional pCofilin ${ }^{+}$puncta found at $30 \mathrm{~s}$ and $15-30 \mathrm{~min}$ post-TBS, and in control slices, were atypically large (data not shown).

Frequency distributions of sizes were generated for PSD95 ${ }^{+}$synapses that were, or were not, colocalized with pPAK immunoreactivity at 2-7 min post-TBS (Fig. $5 a)$. The synapse frequencies are from the sampling zones of eight slices (18,000 synapses total) and are expressed as a fraction of the total population (pPAK positive or negative) for these slices; the labeled synapses associated with $\mathrm{pPAK}^{+}$puncta represented $11 \%$ of the total population of PSD95 $^{+}$structures. Sizes for PSD95 ${ }^{+}$ single-labeled synapses formed a Poisson distribution, whereas those for the double-labeled (PSD95 ${ }^{+} / \mathrm{pPAK}^{+}$) synapses distributed themselves in a more Gaussian manner; the difference in the frequency distributions was highly significant (Kolmogorov-Smirnov $Z, D=0.34$, $p=0.00001)$. Although TBS triggered phosphorylation and the appearance of large PSD95 ${ }^{+}$puncta in a small group of spines, it had no detectable effects on the mean size, or frequency distribution of sizes, within the much larger group of PSD95-labeled profiles (Fig. 5b). This is in accord with physiological and anatomical evidence that LTP is synapse specific and involves changes in a small percentage of the spine population. Comparable results were obtained in a second study using double labeling for pCofilin and PSD95: the PSD95 ${ }^{+}$-only curve followed a Poisson, whereas the $\mathrm{PSD}^{+} / \mathrm{pCofilin}^{+}$ curve was more Gaussian (Fig. 5c) (Kolmogorov-Smirnov $Z, D=0.31, p=$ $0.00001)$. The similarity in the results from the two studies (pPAK and pCofilin) is striking, with the pertinent curves being almost indistinguishable. Finally, we
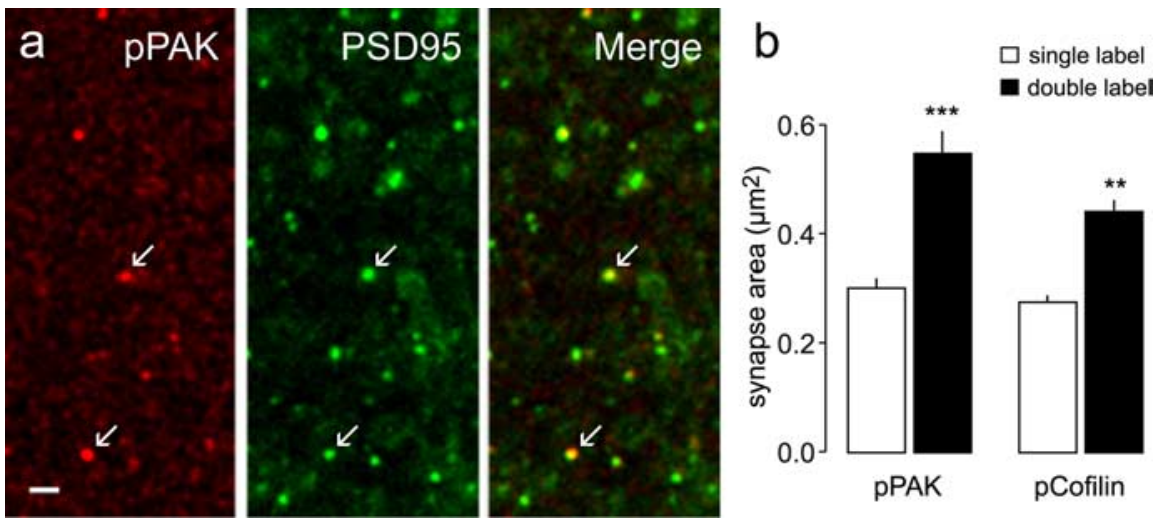

Figure 4. Activation of the PAK/cofilin pathway is associated with large synapses. Slices were collected 2-7 min after delivery of TBS to their Schaffer-commissural afferents. $\boldsymbol{a}$, Micrographs show immunostaining for pCofilin (red), PSD95 (green), and the overlay image (bottom) for a slice harvested 2 min post-TBS. As shown, densely pCofilin-IR puncta (arrows, top) are associated with relatively large PSD95-IR puncta (middle). Scale bar, $1 \mu \mathrm{m}$. $\boldsymbol{b}$, Bar graph summarizes the measured areas of PSD95-IR puncta that were double labeled (black bars) for pPAK immunoreactivity (left) or pCofilin immunoreactivity (right) compared with areas of elements labeled for PSD95 alone (open bars) in the same tissue sections. The differences between sizes of PSD95-IR synapses double labeled for the phosphoproteins or labeled for PSD95 immunoreactivity alone was highly significant $\left({ }^{* *} p<0.01\right.$, *** $p<0.001$, paired $t$ test vs control; $n=8$ slices for both pPAK and p(ofilin).
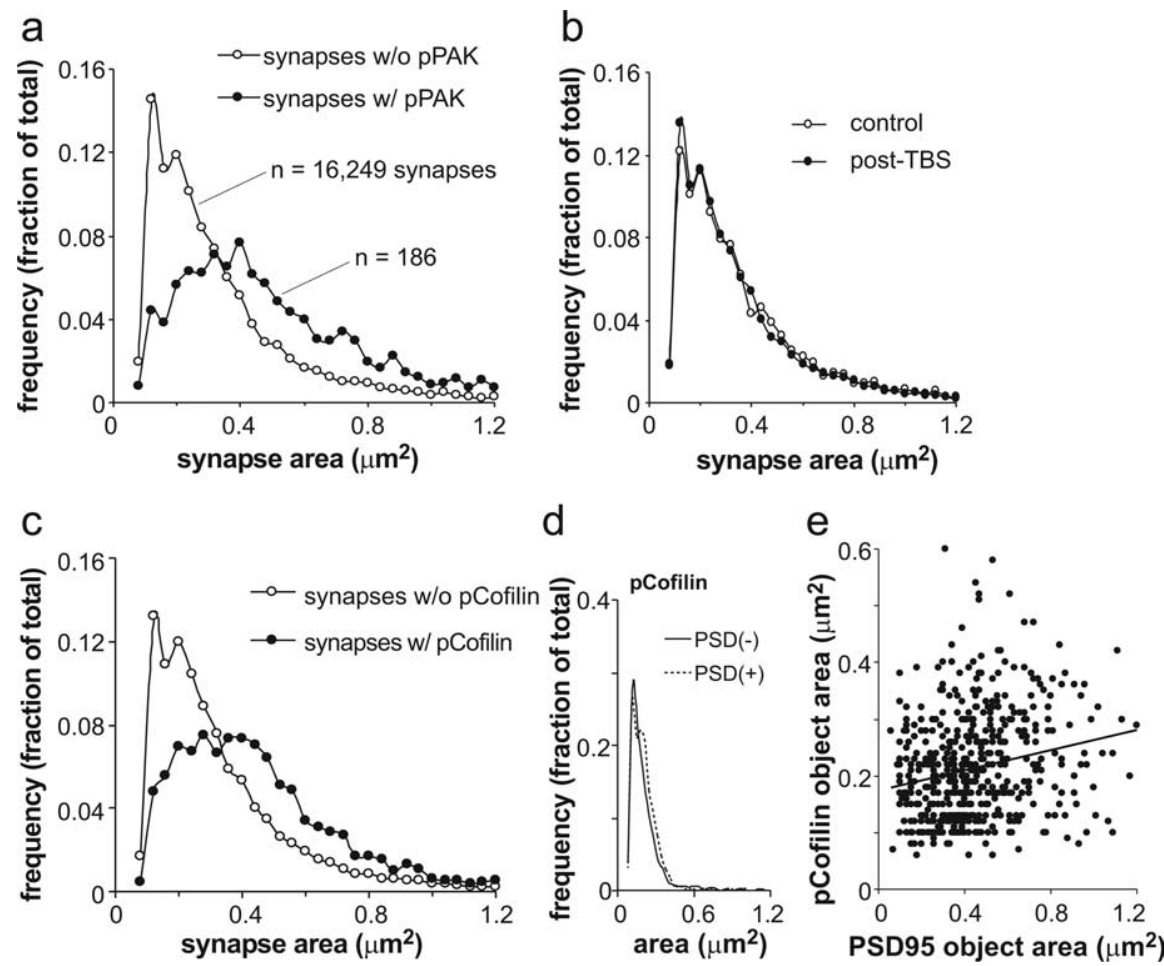

Figure 5. Size distributions differ for synapses located on pPAK- and pCofilin-positive spines compared with synapses that are not associated with a phosphoprotein labeled spine. $\boldsymbol{a}$, Frequency distributions for areas of PSD95-IR synapses with (w/; closed circles) or without (w/0; open circles) associated pPAK immunoreactivity. These measurements were taken from the same tissue sections through slices collected 2-7 min post-TBS. The numbers of synapses used in calculating the distributions are described on the plot. PSD95-IR profiles associated with pPAK immunoreactivity were relatively infrequent. $\boldsymbol{b}$, TBS had no detectable effects on the size distributions for postsynaptic densities that were not situated on pPAK-IR spines. $c$, Frequency distributions for areas of PSD95-IR synapses that were (closed circles) or were not (open circles) located on pCofilin-positive spines in slices collected 2-7 min post-TBS. Note that the results for this experiment replicate those obtained in the pPAK study $(\boldsymbol{a}) . \boldsymbol{d}, A$ comparison of the areas of pCofilin-IR puncta that were (dotted line) or were not (solid line) associated with PSD95 immunostaining. Unlike the reverse case (the sizes of PSD95-IR structures associated with pCofilin shown in c), the areas of pCofilin-IR elements were not affected by the presence or absence of double labeling. $\boldsymbol{e}$, There was no correlation within the group of double-labeled structures between synapse size (PSD95-IR profiles) and the area occupied by pCofilin staining $\left(r^{2}=0.03\right)$. 

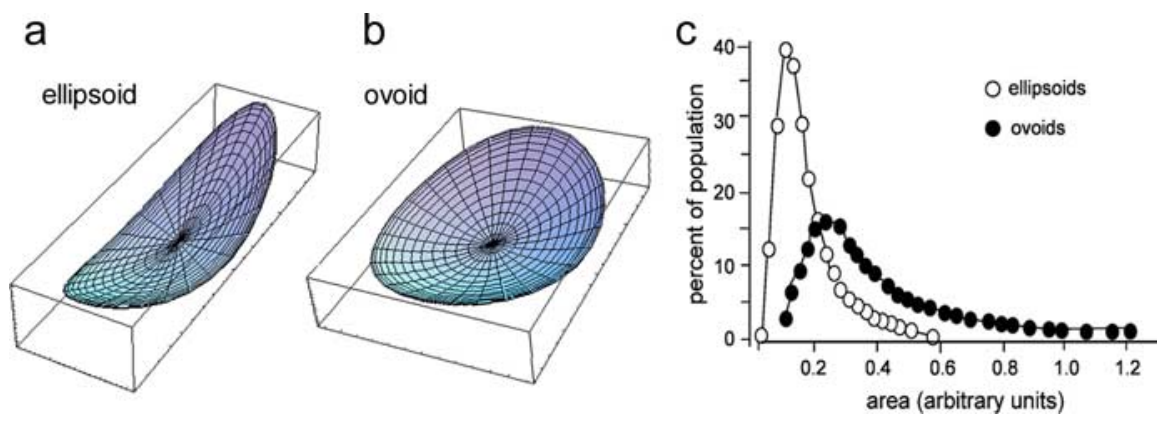

Figure 6. Size distributions of randomly oriented, simulated synapses after projection onto a two-dimensional surface. $\boldsymbol{a}$, Average member of a group of ellipsoid, slightly concave objects used in the simulation. The semi-axes of the ellipsoids were varied according to parameters described in the text so as to produce a population of 20,000 structures that differed in size and shape. $\boldsymbol{b}$, Average member of a group of ovoids used to simulate a second population of synapses. As with the first group, the axes of the ovoids were independently varied so as to produce a collection of objects that varied in size and shape. The mean circumference of the ovoids was the same as that for the ellipsoids. $c$, Distribution of areas of the projected two-dimensional images for the two populations of simulated synapses. Both populations were oriented randomly in a layer that was as thick as the longest simulated synapse and projection was made along the axis perpendicular to the layer. The mean area of the projected ovoids was $60 \%$ greater than that for the projected ellipsoids. The frequency distribution of ellipsoids approximated a Poisson, whereas that for the ovoids was more Gaussian.

tested whether double labeling affected the size (and size distribution) of pPAK- (data not shown) and pCofilinimmunostained elements in the slices used in the above analyses (Fig. $5 d$ ). The area of pCofilin staining was the same in spine-like profiles with and without associated PSD95 immunoreactivity, whereas the frequency distributions of $\mathrm{pCofilin}^{+}$areas were not detectably different for the single- and double-labeled groups. Thus, double labeling by itself does not change the measured size of target structures. As expected from this conclusion, there was no correlation between the areas of PSD95 ${ }^{+}$and pCofilin ${ }^{+}$elements across the population of double-labeled profiles for the 2-7 min groups $\left(r^{2}=0.03\right)$ (Fig. 5e).

In all, then, activation of the PAK/cofilin pathway is associated with a $60-80 \%$ increase in the size of postsynaptic densities and a change in the frequency distribution of their areas. A possible explanation for these two effects is described below.

\section{Simulation of synaptic changes associated with LTP}

A difference in the frequency distribution for sizes, as is evident for the PSD95 ${ }^{+}$synapses on pPAK- or pCofilin-positive puncta versus those not situated on phosphoprotein labeled spines, is suggestive of a difference in the shape of the objects being sampled. We explored this idea with a simulation in which flattened (i.e., $z$-dimension is short), ellipsoid discs were placed at random orientations to the vertical in a three-dimensional layer and then projected from a vertical viewpoint onto a flat surface. The shapes were developed from the standard ellipsoid, which is, in canonical form, as follows:

$$
\frac{x^{2}}{a^{2}}+\frac{y^{2}}{b^{2}}+\frac{z^{2}}{c^{2}}=1,
$$

where $a, b$, and $c$ are the semi-axes of the ellipsoid. For the purpose of evaluating points on the ellipsoid, it is convenient to use a parametric form of Equation 1, which can be given by the mapping $\vec{f}(\theta, \phi): \mathfrak{R}^{2}, \rightarrow \mathfrak{\Re}^{3}$, where

$$
\vec{f}(\theta, \phi)=\left\{\begin{array}{c}
x(\theta, \phi) \\
y(\theta, \phi) \\
z(\theta, \phi)
\end{array}\right\}=\left\{\begin{array}{c}
a \cos (\theta) \sin (\varphi) \\
b \sin (\theta) \sin (\varphi) \\
c \cos (\varphi)
\end{array}\right\},
$$

0 [lteq] $2 \pi$, and 0 [lteq] $\varphi$ [lteq] $\pi$.
To model the curved nature of the synapse, a parabolic function of $x$ and $y$ were added to the $z$ component of Equation 2, as follows:

$$
\begin{aligned}
z^{*}= & d\left((a \cos (\theta) \sin (\phi))^{2}+\right. \\
& \left.(b \sin (\theta) \sin (\phi))^{2}\right)+c \cos (\phi) .
\end{aligned}
$$

Substituting this expression in for the $z$ term in Equation 2 gives the function $\vec{g}(\theta, \varphi)$ where

$$
\vec{g}(\theta, \varphi)=\left\{\begin{array}{c}
x(\theta, \phi) \\
y(\theta, \phi) \\
z^{\star}(\theta, \phi)
\end{array}\right\}
$$

with $z^{\star}$ coming from Equation 3. A typical model of a synapse generated from Equation 4 is shown in Figure $6 a$.

To perform the simulation, the axes values were taken from Gaussian distributions centered at specified means, where the $z$ dimension is held small to achieve shapes that have a flattened appearance. Next, to orient the shapes in space, the rotational transformations about the $x$-, $y$-, and $z$-axes were used:

$$
\begin{aligned}
& R_{x}(\alpha)=\left[\begin{array}{ccc}
1 & 0 & 0 \\
0 & \cos (\alpha) & \sin (\alpha) \\
0 & -\sin (\alpha) & \cos (\alpha)
\end{array}\right], \\
& R_{y}(\beta)=\left[\begin{array}{ccc}
\cos (\beta) & 0 & -\sin (\beta) \\
0 & 1 & 0 \\
\sin (\beta) & 0 & \cos (\beta)
\end{array}\right], \text { and } \\
& R_{z}(\gamma)=\left[\begin{array}{ccc}
\cos (\gamma) & \sin (\gamma) & 0 \\
-\sin (\gamma) & \cos (\gamma) & 0 \\
0 & 0 & 1
\end{array}\right],
\end{aligned}
$$

where the angles $\alpha, \beta$, and $\gamma$ are chosen from uniformly distributed random variables. Points $\vec{p} \varepsilon \Re^{3}$ on a randomly oriented synapse-like shape can then be generated from the following:

$$
\begin{aligned}
& \vec{p}(\theta, \varphi)=R_{x}(\alpha) R_{y}(\beta) R_{z}(\gamma) \vec{g}(\theta, \varphi)= \\
& R_{x}(\alpha) R_{y}(\beta) R_{z}(\gamma)\left[\begin{array}{c}
x(\theta, \phi) \\
y(\theta, \phi) \\
z^{\star}(\theta, \phi)
\end{array}\right] .
\end{aligned}
$$

The locus of points generated by projections from Equation 6 onto the $x-y$ plane is bounded by the curve given by the normal to the object when the $y$-component of the normal is zero. However, because evaluating this curve numerically or obtaining a closed form expression for the curve from Equation 6 is quite complex, a simpler but computationally more expensive approach was taken. In this, a dense series of points were evaluated from applying Equation 6 to the simulated ellipsoid, and these were projected onto a grid in the $x-y$ plane. Elements on this grid behave like pixels on a video monitor in the sense that if enough points are generated on the object, the set of elements in the grid will fill in, and the area of the projected shape can be obtained by simply counting up the filled-in elements.

The areas of the projected shapes, calculated by the means just described, were then measured and plotted as frequency versus size. The projected images are distributed in a Poisson manner 
(Fig. $6 c$ ), with the curve resembling that for unattached synapses (Fig. $5 a, d$ ). This also held when the true distribution (nonprojected and measured in three dimensions) of the objects was measured.

Having found that randomly oriented, highly variable, synapse-like ellipsoids will generate a Poisson distribution, we explored ways in which changing the objects would both increase their mean size and shift their distribution toward a binomial. Figure $6 b$ shows results obtained when the lengths of the axes were adjusted in the direction of an intermediate value, thereby making the objects more oval. In addition, the changes in axes length were constrained by a requirement that circumference of the ovoids equaled that of the ellipsoids. These manipulations caused a $60 \%$ increase in the area of the objects, as measured from the twodimensional projections, and changed their size distribution toward a binomial (Fig. $6 c$ ). Measurements performed in three dimensions produced a comparable result. These results demonstrate that a simple rounding of synapses after activation of the PAK/cofilin system would produce the increase in size and altered frequency distribution of sizes obtained in the present microscopic studies.

\section{Number of pCofilin-positive puncta in control slices is dependent on excitatory transmission}

The occasional presence of $\mathrm{pPAK}^{+}$and $\mathrm{pCofilin}^{+}$puncta with large synapses in control slices points to the possibility that this combination represents a spine "state" that occurs spontaneously in the absence of high-frequency afferent activity. Such events would necessarily be rare with the state having a half-life of a few minutes; otherwise, the labeled profiles would be expected to be much more common. The question then arises as to whether activation of the $\mathrm{PAK} /$ cofilin pathway is a response to randomly occurring combinations of signals impinging on the spine. Perhaps the most likely candidate for a trigger of this kind would be constitutive neurotransmitter release from axon terminals. We tested the idea that glutamatergic transmission might be critical for this phenomenon by incubating slices for $55 \mathrm{~min}$ with $10 \mu \mathrm{M}$ DNQX, an antagonist of AMPA-type glutamate receptors. Stimulation and recording arrangements were as in previous experiments; measures of evoked responses from str. radiatum (Fig. $7 a, b)$ indicate that transmission was blocked for $>30$ min before fixing the slice. It was evident from survey micrographs (Fig. 7c) that pCofilin $^{+}$puncta were much less frequent within the physiological recording zone from DNQX-treated slices than was the case for matched controls. Quantitatively, the AMPA receptor antagonist reduced the number of pCofilin ${ }^{+}$puncta by $>50 \%$ (two-tailed paired $t$ test, $p=0.003$ ).

\section{Discussion}

Past work showed that a train of 10 theta bursts causes a marked increase in the number of spines containing high concentrations of F-actin (Lin et al., 2005; Kramar et al., 2006); this effect is eliminated by latrunculin A (Rex et al., 2007), confirming that the stimulation triggered an intense but localized bout of actin polymerization. The actin effect was completely blocked by neutralizing antibodies against $\beta 1$ family integrins (Kramar et al., 2006) or by a scavenger of BDNF (Rex et al., 2007). This suggests that theta bursts, which release BDNF (Balkowiec and Katz, 2002; Aicardi et al., 2004), engage integrin/trophic factor machinery of a type used by many cell types to translate extracellular signals into morphological changes and altered gene expression. The potent influence of integrins and the TrkB receptor of BDNF over the cytoskeleton involves the Rho family (Geiger et al., 2002; Brakebusch and Fassler, 2003; DeMali et al., 2003; Gehler et al., 2004; Juliano et al., 2004; Wiesner et al., 2005; Chen et al., 2006; Legate et al., 2006), a group of GTPases that collectively act on nucleation and elongation factors as well as on the PAK/cofilin regulatory pathway (Ridley, 2001). The present studies provide the first evidence that this link between surface receptors and the cytoskeleton is activated in mature dendritic spines by thetaburst stimulation. Single theta trains caused severalfold increases in the numbers of spines containing dense concentrations of pPAK and cofilin in the dendritic zones innervated by the stimulated afferents, but not in the adjacent, more distal, dendritic field. Moreover, the time course for the onset of phosphorylation aligned with that described previously for theta-induced actin polymerization (Kramar et al., 2006). It appears, then, that synaptic activity engages known signaling pathways that are used by integrins and BDNF receptors to alter actin dynamics within adult dendritic spines.

The increase in spines with evidence of PAK/cofilin activation agreed reasonably well with estimates of the number of synapses in which LTP occurs. Recordings from pairs of pyramidal cells in adult hippocampal slices suggest that a minimum of two contacts generates a mean evoked (one cell to one cell) EPSP (Debanne et al., 1996; Pavlidis and Madison, 1999) that is $<10 \%$ of the amplitude of the composite EPSP typically used in LTP experiments. If we assume that $\sim 25$ contacts per neuron are involved in the production of the stable (over time) and robust responses used in the present experiments, and take into account the fact that two converging inputs were used, we can estimate that a minimum of 50 synapses per neuron were activated by TBS. Anatomical work indicates that a typical CA1 neuron has 15,000 spines within the entire str. radiatum (Ishizuka et al., 1995; Megias et al., 2001), suggesting that $\sim 7500$ spines (per neuron) are found in the proximal half of the lamina containing the activated synaptic population. However, it is unlikely that the greater part of the dendritic 
tree is present within a $350-\mu \mathrm{m}$-thick slice. Assuming that onethird of the tree is retained, an estimated 2500 synapses would be found within the target dendritic zone. Combining these values, we can estimate that LTP in the present studies occurred in $\sim 2 \%$ of the spine population on a given neuron. This estimate is close to the calculated percentage $(1.3 \%)$ of the total spine population $\left(\sim 250\right.$ synapses/100 $\mu^{3}{ }^{3}$ ) (Sorra and Harris, 1998; Scheff et al., 2005) that exhibited a theta stimulation-induced increase in PAK phosphorylation; this value is likely to be an underestimate because of incomplete labeling with the immunostaining technique.

The discovery that the induction of LTP is associated with phosphorylation of PAK and cofilin opened the way to a test of the long-standing hypothesis that the potentiation effect involves a change in synaptic morphology (Lynch and Baudry, 1984; Yuste and Bonhoeffer, 2001). Early electron microscopic studies showed that the distribution of synaptic lengths changed after induction of LTP (Lee et al., 1979, 1980), a result interpreted as suggesting that a group of rounder profiles had been added to the population. Subsequent studies found that synapses were larger after induction or became split by intervening spine membrane (Jones and Calverley, 1991; Toni et al., 2001; Ostroff et al., 2002; Harris et al., 2003; Lang et al., 2004). Together these findings argue that LTP induction changes synaptic morphology, although no clear consensus as to the nature of the change has emerged. The shape change (of PSD95-IR synapses) observed in the present studies did not involve a simple expansion (i.e., multiplication of size or staining intensity) because the frequency distribution of synaptic areas for synapses on PPAK- or pCofilinpositive spines was very different from that for synapses not associated with the phosphoproteins. A simulation of randomly oriented synapse-like profiles confirmed the suspicion that the observed distributions and greater size could arise if stretched ellipsoids were converted into ovoids. Notably, the transformations involved in the modeling study did not involve any change in synaptic circumference, a result suggesting that a simple rounding of the structures is sufficient to produce the observed increase in area.

Unexpectedly, the few pCofilin-positive spines found in control (no TBS) slices had unusually large synapses; moreover, the frequency distribution of synapse areas showed the same difference between pCofilin-positive and -negative spines as that found after induction of LTP. Given that phosphorylation is a transient event, it seems inevitable that the increase in pCofilinpositive spines occurred sometime in the few minutes before the moment that the slices were collected and fixed. Phosphorylation, in other words, appears to be a spontaneous event. This conclusion implies that the large synapses found on the $\mathrm{PPAK}^{+}$ or pCofilin ${ }^{+}$spines also developed spontaneously and are transient. An alternative hypothesis would be that there is a very small population of spines with large synapses and that the PAK/cofilin pathway is continuously activated in these. However, blocking glutamatergic transmission with DNQX, in otherwise untreated slices, markedly reduced the number of spines with dense concentrations of phosphorylated cofilin and attendant large synapses. These results reinforce the conclusion that activation of the $\mathrm{PAK} /$ cofilin pathway occurs spontaneously in slices and is associated with the transient emergence of large, presumably ovoid synapses.

Assuming that larger synapses are indicative of enhanced transmission, the above argument implies that the potentiated state is a stochastic event, with a very low probability obtained under resting conditions and a high probability of occurring in spines that received theta-burst stimulation. This would require a low threshold for potentiation, and there are reasons to think that this is the case. For example, using two inputs to the same dendritic targets, it is possible to generate a measurable degree of LTP with a single theta burst (four EPSPs) by activating the other input $200 \mathrm{~ms}$ earlier (Larson et al., 1986). It will be of interest in future studies to determine whether the magnitude of the synapse size change is affected by the amount of theta-burst stimulation used. If the number of bursts, or repetition of trains, simply increases the number of spines with large synapses, but does not influence the size distribution of those synapses, it is likely to be the case that LTP is a quantum effect.

The idea that LTP has a low threshold relates only to induction and expression: it would seem that stabilization (consolidation) is another matter. Given the small number of large synapses found in control slices, it must be the case that those developing along with the intermittent, spontaneous activation of the PAK/ cofilin pathway quickly revert to a previous, smaller synapse state. It thus appears that the phosphorylation events are not sufficient to trigger the full range of processes that make persistent changes in synaptic morphology. As noted above, actin polymerization and LTP are readily reversed during the first several minutes post-TBS but then become resistant to disruption. The processes responsible for this acquired resistance could thus constitute the high-threshold step in the production of LTP.

\section{References}

Aicardi G, Argilli E, Cappello S, Santi S, Riccio M, Thoenen H, Canossa M (2004) Induction of long-term potentiation and depression is reflected by corresponding changes in secretion of endogenous brain-derived neurotrophic factor. Proc Natl Acad Sci USA 101:15788-15792.

Aoki C, Miko I, Oviedo H, Mikeladze-Dvali T, Alexandre L, Sweeney N, Bredt DS (2001) Electron microscopic immunocytochemical detection of PSD-95, PSD-93, SAP-102, and SAP-97 at postsynaptic, presynaptic, and nonsynaptic sites of adult and neonatal rat visual cortex. Synapse 40:239-257.

Balkowiec A, Katz DM (2002) Cellular mechanisms regulating activitydependent release of native brain-derived neurotrophic factor from hippocampal neurons. J Neurosci 22:10399-10407.

Boda B, Alberi S, Nikonenko I, Node-Langlois R, Jourdain P, Moosmayer M, Parisi-Jourdain L, Muller D (2004) The mental retardation protein PAK3 contributes to synapse formation and plasticity in hippocampus. J Neurosci 24:10816-10825.

Boda B, Nikonenko I, Alberi S, Muller D (2006) Central nervous system functions of PAK protein family: from spine morphogenesis to mental retardation. Mol Neurobiol 34:67-80.

Brakebusch C, Fassler R (2003) The integrin-actin connection, an eternal love affair. EMBO J 22:2324-2333.

Carlisle HJ, Kennedy MB (2005) Spine architecture and synaptic plasticity. Trends Neurosci 28:182-187.

Chen TJ, Gehler S, Shaw AE, Bamburg JR, Letourneau PC (2006) Cdc42 participates in the regulation of $\mathrm{ADF} /$ cofilin and retinal growth cone filopodia by brain derived neurotrophic factor. J Neurobiol 66:103-114.

Chong C, Tan L, Lim L, Manser E (2001) The mechanism of PAK activation. Autophosphorylation events in both regulatory and kinase domains control activity. J Biol Chem 276:17347-17353.

Debanne D, Gahwiler BH, Thompson SM (1996) Cooperative interactions in the induction of long-term potentiation and depression of synaptic excitation between hippocampal CA3-CA1 cell pairs in vitro. Proc Natl Acad Sci USA 93:11225-11230.

DeMali KA, Wennerberg K, Burridge K (2003) Integrin signaling to the actin cytoskeleton. Curr Opin Cell Biol 15:572-582.

Fiala JC, Harris KM (2001) Extending unbiased stereology of brain ultrastructure to three-dimensional volumes. J Am Med Inform Assoc 8:1-16.

Fifkova E, Van Harreveld A (1977) Long-lasting morphological changes in dendritic spines of dentate granular cells following stimulation of the entorhinal area. J Neurocytol 6:211-230.

Fischer M, Kaech S, Knutti D, Matus A (1998) Rapid actin-based plasticity in dendritic spines. Neuron 20:847-854. 
Fukazawa Y, Saitoh Y, Ozawa F, Ohta Y, Mizuno K, Inokuchi K (2003) Hippocampal LTP is accompanied by enhanced F-actin content within the dendritic spine that is essential for late LTP maintenance in vivo. Neuron 38:447-460.

Ganeshina O, Berry RW, Petralia RS, Nicholson DA, Geinisman Y (2004) Differences in the expression of AMPA and NMDA receptors between axospinous perforated and nonperforated synapses are related to the configuration and size of postsynaptic densities. J Comp Neurol 468:86-95.

Gehler S, Shaw AE, Sarmiere PD, Bamburg JR, Letourneau PC (2004) Brain-derived neurotrophic factor regulation of retinal growth cone filopodial dynamics is mediated through actin depolymerizing factor/ cofilin. J Neurosci 24:10741-10749.

Geiger B, Bershadsky A, Pankov R, Yamada KM (2002) Transmembrane extracellular matrix-cytoskeleton crosstalk. Nat Rev Mol Cell Biol 2:793-805.

Harris KM, Fiala JC, Ostroff L (2003) Structural changes at dendritic spine synapses during long-term potentiation. Philos Trans R Soc Lond B Biol Sci 358:745-748.

Hayashi ML, Choi SY, Rao BS, Jung HY, Lee HK, Zhang D, Chattarji S, Kirkwood A, Tonegawa S (2004) Altered cortical synaptic morphology and impaired memory consolidation in forebrain-specific dominantnegative PAK transgenic mice. Neuron 42:773-787.

Hunt CA, Schenker LJ, Kennedy MB (1996) PSD-95 is associated with the postsynaptic density and not with the presynaptic membrane at forebrain synapses. J Neurosci 16:1380-1388.

Ishizuka N, Cowan WM, Amaral DG (1995) A quantitative analysis of the dendritic organization of pyramidal cells in the rat hippocampus. J Comp Neurol 362:17-45.

Jain AK (1984) Fundamentals of digital image processing. Englewood Cliffs, NJ: Prentice Hall.

Jones DG, Calverley RK (1991) Perforated and non-perforated synapses in rat neocortex: three-dimensional reconstructions. Brain Res 556:247-258.

Juliano RL, Reddig P, Alahari S, Edin M, Howe A, Aplin A (2004) Integrin regulation of cell signalling and motility. Biochem Soc Trans 32:443-446.

Kramar EA, Lin B, Rex CS, Gall CM, Lynch G (2006) Integrin-driven actin polymerization consolidates long-term potentiation. Proc Natl Acad Sci USA 103:5579-5584.

Lang C, Barco A, Zablow L, Kandel ER, Siegelbaum SA, Zakharenko SS (2004) Transient expansion of synaptically connected dendritic spines upon induction of hippocampal long-term potentiation. Proc Natl Acad Sci USA 101:16665-16670.

Larson J, Wong D, Lynch G (1986) Patterned stimulation at the theta frequency is optimal for the induction of hippocampal long-term potentiation. Brain Res 368:347-350.

Lee K, Oliver M, Schottler F, Creager R, Lynch G (1979) Ultrastructural effects of repetitive synaptic stimulation in the hippocampal slice preparation: a preliminary report. Exp Neurol 65:478-480.

Lee KS, Schottler F, Oliver M, Lynch G (1980) Brief bursts of highfrequency stimulation produce two types of structural change in rat hippocampus. J Neurophysiol 44:247-258.

Legate KR, Montanez E, Kudlacek O, Fassler R (2006) ILK, PINCH and parvin: the tIPP of integrin signalling. Nat Rev Mol Cell Biol 7:20-31.

Lin B, Kramar EA, Bi X, Brucher FA, Gall CM, Lynch G (2005) Theta stimulation polymerizes actin in dendritic spines of hippocampus. J Neurosci 25:2062-2069.

Lynch G, Baudry M (1984) The biochemistry of memory: a new and specific hypothesis. Science 224:1057-1063.

Matus A, Brinkhaus H, Wagner U (2000) Actin dynamics in dendritic spines: a form of regulated plasticity at excitatory synapses. Hippocampus 10:555-560.

Megias M, Emri Z, Freund TF, Gulyas AI (2001) Total number and distribution of inhibitory and excitatory synapses on hippocampal CA1 pyramidal cells. Neuroscience 102:527-540.

Muller D, Toni N, Buchs PA (2000) Spine changes associated with longterm potentiation. Hippocampus 10:596-604.

Nagerl UV, Eberhorn N, Cambridge SB, Bonhoeffer T (2004) Bidirectional activity-dependent morphological plasticity in hippocampal neurons. Neuron 44:759-767.
Nusser Z, Lujan R, Laube G, Roberts JD, Molnar E, Somogyi P (1998) Cell type and pathway dependence of synaptic AMPA receptor number and variability in the hippocampus. Neuron 21:545-559.

Okamoto K, Nagai T, Miyawaki A, Hayashi Y (2004) Rapid and persistent modulation of actin dynamics regulates postsynaptic reorganization underlying bidirectional plasticity. Nat Neurosci 7:1104-1112.

Ostroff LE, Fiala JC, Allwardt B, Harris KM (2002) Polyribosomes redistribute from dendritic shafts into spines with enlarged synapses during LTP in developing rat hippocampal slices. Neuron 35:535-545.

Pavlidis P, Madison DV (1999) Synaptic transmission in pair recordings from CA3 pyramidal cells in organotypic culture. J Neurophysiol 81:2787-2797.

Penzes P, Beeser A, Chernoff J, Schiller MR, Eipper BA, Mains RE, Huganir RL (2003) Rapid induction of dendritic spine morphogenesis by transsynaptic ephrinB-EphB receptor activation of the Rho-GEF kalirin. Neuron 37:263-274.

Petersen JD, Chen X, Vinade L, Dosemeci A, Lisman JE, Reese TS (2003) Distribution of postsynaptic density (PSD)-95 and $\mathrm{Ca}^{2+} /$ calmodulindependent protein kinase II at the PSD. J Neurosci 23:11270-11278.

Racca C, Stephenson FA, Streit P, Roberts JD, Somogyi P (2000) NMDA receptor content of synapses in stratum radiatum of the hippocampal CA1 area. J Neurosci 20:2512-2522.

Racz B, Weinberg RJ (2006) Spatial organization of cofilin in dendritic spines. Neuroscience 138:447-456.

Rex CS, Kramar EA, Colgin LL, Lin B, Gall CM, Lynch G (2005) Long-term potentiation is impaired in middle-aged rats: regional specificity and reversal by adenosine receptor antagonists. J Neurosci 25:5956-5966.

Rex CS, Lauterborn JC, Lin CY, Kramar EA, Rogers GA, Gall CM, Lynch G (2006) Restoration of long-term potentiation in middle-aged hippocampus after induction of brain-derived neurotrophic factor. J Neurophysiol 96:677-685.

Rex CS, Lin CY, Kramar EA, Chen LY, Gall CM, Lynch G (2007) Brainderived neurotrophic factor promotes long-term potentiation-related cytoskeletal changes in adult hippocampus. J Neurosci 27:3017-3029.

Ridley AJ (2001) Rho GTPases and cell migration. J Cell Sci 114:2713-2722.

Sassoe-Pognetto M, Utvik JK, Camoletto P, Watanabe M, Stephenson FA, Bredt DS, Ottersen OP (2003) Organization of postsynaptic density proteins and glutamate receptors in axodendritic and dendrodendritic synapses of the rat olfactory bulb. J Comp Neurol 463:237-248.

Scheff SW, Price DA, Hicks RR, Baldwin SA, Robinson S, Brackney C (2005) Synaptogenesis in the hippocampal CA1 field following traumatic brain injury. J Neurotrauma 22:719-732.

Shimono K, Kubota D, Brucher F, Taketani M, Lynch G (2002) Asymmetrical distribution of the Schaffer projections within the apical dendrites of hippocampal field CA1. Brain Res 950:279-287.

Sorra KE, Harris KM (1998) Stability in synapse number and size at $2 \mathrm{hr}$ after long-term potentiation in hippocampal area CA1. J Neurosci 18:658-671.

Soule J, Messaoudi E, Bramham CR (2006) Brain-derived neurotrophic factor and control of synaptic consolidation in the adult brain. Biochem Soc Trans 34:600-604.

Takumi Y, Ramirez-Leon V, Laake P, Rinvik E, Ottersen OP (1999) Different modes of expression of AMPA and NMDA receptors in hippocampal synapses. Nat Neurosci 2:618-624.

Toni N, Buchs PA, Nikonenko I, Povilaitite P, Parisi L, Muller D (2001) Remodeling of synaptic membranes after induction of long-term potentiation. J Neurosci 21:6245-6251.

Valtschanoff JG, Weinberg RJ (2001) Laminar organization of the NMDA receptor complex within the postsynaptic density. J Neurosci 21:1211-1217.

Wiesner S, Legate KR, Fassler R (2005) Integrin-actin interactions. Cell Mol Life Sci 62:1081-1099.

Yuste R, Bonhoeffer T (2001) Morphological changes in dendritic spines associated with long-term synaptic plasticity. Annu Rev Neurosci 24:1071-1089.

Zhou Q, Homma KJ, Poo MM (2004) Shrinkage of dendritic spines associated with long-term depression of hippocampal synapses. Neuron 44:749-757. 